\title{
Sweet's Syndrome: One Disease, Multiple Faces
}

\author{
Yusuf Kasirye, MD; Rebecca S. Danhof, MPH; Narendranath Epperla, MD; \\ and Romel J. Garcia-Montilla, MD, PhD
}

\section{Case Presentations}

\section{Case 1}

A man, aged 48 years, with a history of myelodysplastic syndrome, post-decitabine chemotherapy was admitted with a fever, skin rash, and pancytopenia. Physical examination showed multiple painless, non-itchy, erythematous, maculopapular skin lesions on the dorsum of both hands (figure 1A). Broad spectrum antibiotics, antifungals, and granulocyte colony-stimulating factor (G-CSF) therapy were started. The septic workup came back negative, yet the patient continued having low-grade fever with new skin lesions. Skin biopsy revealed diffuse dermal neutrophilic infiltrate (figure 1B), consistent with Sweet's syndrome. Prednisone $(1 \mathrm{mg} / \mathrm{kg} /$ day $)$ was started and antibiotics were de-escalated. The skin lesions regressed, and the fever resolved in two days. Triggering factors were thought to be decitabine, myelodysplastic syndrome, and G-CSF therapy. He was discharged on oral prednisone.
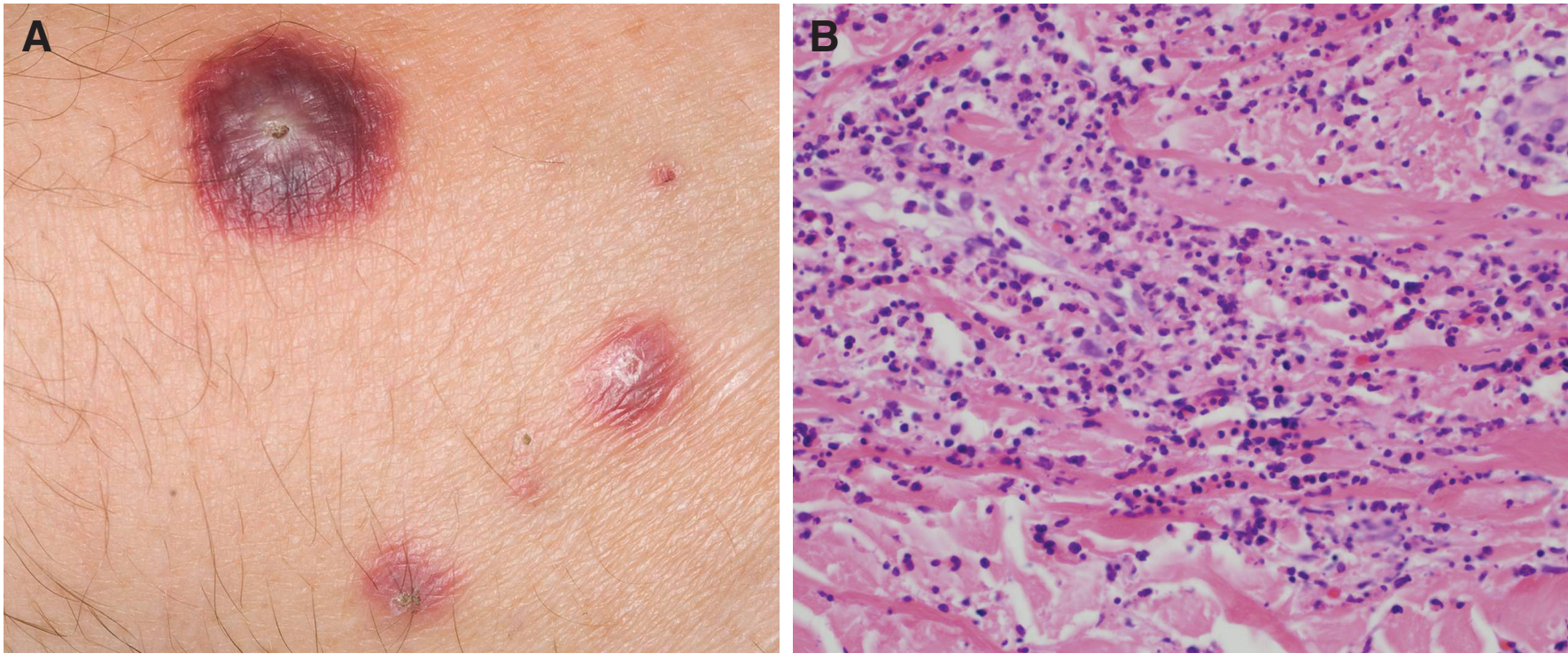

Figure 1. (A) Examples of multiple non-tender, erythematous papules (less than $1 \mathrm{~cm}$ ), with a pseudo-bullous aspect on the dorsum of one hand. (B) Diffuse dermal neutrophilic infiltrate with papillary edema, consistent with Sweet's syndrome. Polymorphonuclear cells can also be noted (Hematoxylin \& Eosin staining, $\times 40$ ).

Corresponding Author:

Yusuf Kasirye, MD

Department of Internal Medicine

Marshfield Clinic

1000 North Oak Ave

Marshfield, WI 54449 USA

Tel: 7 I 5-387-5537

Fax: 7|5-389-5757

Email: kasirye.yusuf@marshfieldclinic.org
Keywords: Sweet's syndrome; Skin rash; Myelodysplastic syndrome; Neutrophilic dermatosis

Received: April 7, 2011

Revised: June 8, 2011

Accepted: June 15, 2011

doi: $10.3121 / \mathrm{cmr} .2011 .1012$

The Aperture, like the opening in the lens of a microscope that allows light to pass through, is a forum for art, humor, and images that provides a portal for new or different views of medicine and research. 

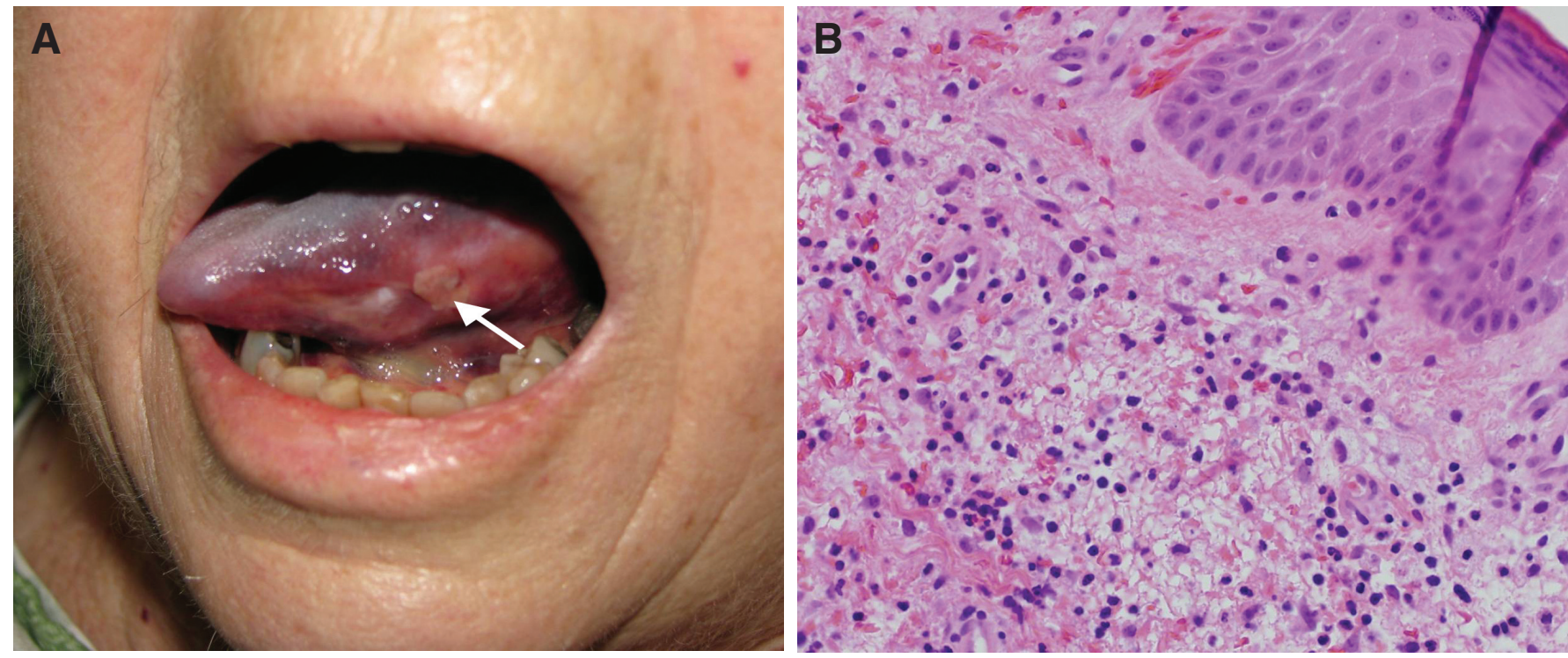

Figure 2. (A) Evidence of macroglossia and oral ulcers (arrow). (B) Skin biopsy showing diffuse dermal neutrophilic infiltrate, papillary edema of both the dermis and epidermis, consistent with Sweet's syndrome (Hematoxylin \& Eosin staining, $\times 40)$.

Case 2

A woman, aged 74 years, with a recent diagnosis of myelodysplastic syndrome and Sweet's syndrome was admitted with acute onset of tongue swelling, painful oral ulcers, fever, and a new skin rash. She was on a tapering dose of prednisone. Physical examination revealed oral ulcers, macroglossia (figure 2A) (with no airway compromise) and an ecchymotic, violaceous skin rash on her trunk. Skin biopsy revealed neutrophilic dermatosis consistent with Sweet's syndrome (figure 2B). Bone marrow biopsy revealed myelodysplastic syndrome transformation to acute myelogenous leukemia. She was started on azacitidine. Control of Sweet's syndrome was achieved with a combination of high dose prednisone and supersaturated potassium iodide. Her symptoms resolved, and she was discharged on oral medications.

\section{Discussion}

Sweet's syndrome, or acute febrile neutrophilic dermatosis, is an unusual dermatologic disorder first described in $1964 .{ }^{1-3}$ It is divided into three categories: idiopathic (classical), druginduced, and malignancy-associated, although the exact cause within any category is not always known. Idiopathic Sweet's syndrome predominantly affects middle-aged females (with a recurrence of up to $50 \%$ ) and could be associated with infections, inflammatory diseases, or pregnancy. Malignancyassociated Sweet's syndrome accounts for approximately $20 \%$ of Sweet's syndrome cases and is commonly associated with myeloproliferative disorders and, to a lesser extent, solid tumors. Drug-induced Sweet's syndrome is an uncommon reaction to medications including G-CSF, non-steroidal antiinflammatory drugs, and decitabine. ${ }^{3-5}$

Fever with skin rash involving the face, trunk, and extremities is the usual presentation of Sweet's syndrome. In its classic form, the lesions are tender, erythematous plaques or papules with a pseudo-bullous or vesiculous component. Systemic manifestations involve mucosal, renal, cardiac, pulmonary, and central nervous systems. Macroglossia, as seen in our second case, can be a herald for myelodysplastic syndrome transformation to secondary acute myelogenous leukemia. ${ }^{4,6,7}$ Although the pathogenesis remains unknown, Sweet's syndrome is thought to bean immune-mediated hypersensitivity reaction to infectious, inflammatory, drug, or tumor cell antigens. Cytokines, dermal dendrocytes, and auto-antibodies might also have a role. ${ }^{2,3,8}$

Although the diagnostic criteria have evolved over time to include more clinical aspects; ${ }^{9}$ the original components, as described by Sweets et al, ${ }^{1,10}$ have remained the key to confirming the diagnosis. The original criteria involve fever, peripheral leukocytosis, tender erythematous plaques, and a diffuse dermal neutrophilic infiltration. Both of our patients did meet these criteria. First line treatment is corticosteroids. Prednisone $(1 \mathrm{mg} / \mathrm{kg} /$ day) can be started and tapered off in two to six weeks according to clinical response. Alternative therapies include supersaturated potassium iodide, rifampin, clofazimine, cyclosporine, dapsone, colchicine, indomethacin, and more recently, tumor necrosis factor antagonists. ${ }^{2,4}$ Treatment of the underlying cause is vital to resolution of this condition. It should also be noted that some cases do resolve spontaneously.

\section{Conclusion}

Given the diverse associations and manifestations of Sweet's syndrome, it should always be considered as a differential diagnosis in patients with an acute febrile illness and skin rash. A timely diagnosis allows the physician to begin appropriate treatment as well as the ability to manage the underlying cause. 


\section{Acknowledgements}

The authors thank Jonathan Cutlan, MD and David Kim, MD, $\mathrm{PhD}$ of the Marshfield Clinic Division of Laboratory Medicine for providing the pathology images. They also thank Thomas McIntee, MD and Clayton $\mathrm{B}$. Green, $\mathrm{MD}, \mathrm{PhD}$ of the Marshfield Clinic Department of Dermatology. The authors further thank the Marshfield Clinic Research Foundation's Office of Scientific Writing and Publication for assistance in the preparation of this manuscript.

\section{References}

1. Sweet RD. An acute febrile neutrophilic dermatosis. Br J Dermatol 1964;76:349-356.

2. Yi S, Bhate C, Schwartz RA. Sweet's syndrome: an update and review. G Ital Dermatol Venereol 2009;144:603-612.

3. Abbas O, Kibbi AG, Rubeiz N. Sweet's syndrome: retrospective study of clinical and histologic features of 44 cases from a tertiary care center. Int J Dermatol 2010;49:1244-1249.

4. Cohen PR. Sweet's syndrome--a comprehensive review of an acute febrile neutrophilic dermatosis. Orphanet J Rare Dis 2007;2:34.

5. Alencar C, Abramowtiz M, Parekh S, Braunshweig I, Jacobson M, Silverman L, Verma A. Atypical presentations of Sweet's syndrome in patients with MDS/AML receiving combinations of hypomethylating agents with histone deacetylase inhibitors. Am J Hematol 2009;84:688-689.

6. Cohen PR, Kurzrock R. Extracutaneous manifestations of Sweet's syndrome: steroid-responsive culture-negative pulmonary lesions. Am Rev Respir Dis 1992;146:269.

7. Bamelis M, Boyden B, Sente F, Madoe V. Sweet's syndrome and acute myelogenous leukemia in a patient who presented with a sudden massive swelling of the tongue. Dermatology 1995;190:335-337.

8. Reuss-Borst MA, Pawelec G, Saal JG, Horny HP, Müller CA, Waller HD. Sweet's syndrome associated with myelodysplasia: possible role of cytokines in the pathogenesis of the disease. Br J Haematol 1993;84:356-358.

9. von den Driesch P. Sweet's syndrome (acute febrile neutrophilic dermatosis) Am Acad Dermatol 1994;31:535-556; quiz 557-560.

10. Genders RE, Vermeer MH, van Doorn R. Erythematous and purpuric plaques of the face and scalp in Sweet syndrome. Br J Haematol 2011;154:159.

\section{Author Affiliations}

Yusuf Kasirye, MD*; Rebecca S. Danhof, $\mathrm{MPH}^{\dagger}$;

Narendranath Epperla, MD*; and

Romel J. Garcia-Montilla, MD, PhDt

*Department of Internal Medicine, Marshfield Clinic, 1000 N Oak Ave, Marshfield, WI USA

tUniversity of Wisconsin School of Medicine \& Public

Health, 750 Highland Ave, Madison, WI, USA

$¥$ Department of Hospital Medicine, Marshfield Clinic, 1000 N Oak Ave, Marshfield, WI USA 